\title{
Rat cardiovascular responses to whole body suspension: head-down and non-head-down tilt
}

\author{
X. J. MUSACCHIA, JOSEPH M. STEFFEN, AND JUDY DOMBROWSKI \\ Departments of Physiology and Biophysics and Biology, University of Louisville, Louisville, Kentucky 40292
}

\begin{abstract}
Musacchia, X. J., Joseph M. STEFFen, AND Judy DomBROWSKI. Rat cardiovascular responses to whole body suspension: head-down and non-head-down tilt. J. Appl. Physiol. 73(4): 1504-1509, 1992.-The rat whole body suspension technique mimics responses seen during exposure to microgravity and was evaluated as a model for cardiovascular responses with two series of experiments. In one series, changes were monitored in chronically catheterized rats during 7 days of head-down tilt (HDT) or non-head-down tilt (N-HDT) and after several hours of recovery. Elevations of mean arterial (MAP), systolic, and diastolic pressures of $\sim 20 \%(P<0.05)$ in HDT rats began as early as day 1 and were maintained for the duration of suspension. Pulse pressures were relatively unaffected, but heart rates were elevated $\sim 10 \%$. During postsuspension ( $2-7 \mathrm{~h}$ ), most cardiovascular parameters returned to presuspension levels. $\mathrm{N}$ HDT rats exhibited elevations chiefly on days 3 and 7 . In the second series, blood pressure was monitored in 1- and 3-day HDT and N-HDT rats to evaluate responses to rapid head-up tilt. MAP, systolic and diastolic pressures, and HR were elevated $(P<0.05)$ in HDT and N-HDT rats during head-up tilt after 1 day of suspension, while pulse pressures remained unchanged. HDT rats exhibited elevated pretilt MAP and failed to respond to rapid head-up tilt with further increase of MAP on day 3 , indicating some degree of deconditioning. The whole body suspended rat may be useful as a model to better understand responses of rats exposed to microgravity.
\end{abstract}

mean arterial blood pressure; systolic blood pressure; diastolic blood pressure; pulse pressure; heart rate; weightlessness; microgravity

EXPOSURE of humans to microgravity is known to result in cardiovascular changes. These include a transient initial increase in cardiac volume and cardiac output that recovers within several days (1). In Shuttle astronauts, cardiovascular deconditioning has been noted as elevated basal and passive tilt-induced heart rates, increased basal mean arterial pressure (MAP), and a tiltinduced fall in MAP (2). On exposure to microgravity, there is a redistribution of body fluids and an increase in central blood volume and pressure (1). The head-downtilted (HDT) rat has been utilized as an animal model to simulate the effects of microgravity on the cardiovascular system and fluid redistribution $(5,7,12,15,17)$. In the rat, HDT is known to induce cephalad fluid shifts (5) with concurrent diuresis, natriuresis, and kaliuresis (3).

Preliminary studies of HDT rats $(9,10)$ indicated elevated MAP, systolic pressure (SP), and diastolic pressure (DP). Popovic (13) found that HDT rats initially had elevated right atrial pressures that returned to base- line after 2 days of suspension. Shellock et al. (15) reported a direct relationship between the increase in central venous pressure and the degree of tilt $\left(20\right.$ and $\left.45^{\circ}\right)$ in HDT rats during a $24-\mathrm{h}$ period. Previous observations of blood pressure changes in the peripheral vascular system are inconsistent, with results suggesting no significant change in MAP (16), a transitory elevation (17), or persistent elevation in MAP during the first 3 days of suspension (9). An examination of the literature indicates that pressure measurements in suspended rats have been made with various techniques, with different methods of suspension (including a lack of consistency in the angle of HDT), and over different periods of HDT (acute 24-h periods and chronic periods of several days). It is likely that variations in published results reflect these different experimental protocols.

The first 2 days of suspension appear to be a period of stress and adjustment. Thus we considered it relevant to assess blood pressure and heart rate (HR) responses during the first 3 days of suspension. Our objectives in this present study were 1) to ascertain $\mathrm{HR}$ and arterial pressure responses in rats during a 7-day period of HDT or non-head-down tilt (N-HDT) and after removal from whole body suspension (WBS) and 2) to determine whether the rat exhibits indications of orthostatic intolerance after rapid reversal of the HDT posture during the first 3 days of suspension. An overall goal of these experiments was to examine further the versatility of the WBS system specifically as a ground-based model for cardiovascular effects of microgravity.

\section{MATERIALS AND METHODS}

Male Sprague-Dawley rats in both series of experiments were suspended in a WBS harness (8) in either an HDT $\left(20^{\circ}\right)$ or an N-HDT position. N-HDT rats are animals suspended in harnesses like those of HDT rats except in a horizontal orientation. The animal room was on a 12:12-h light-dark cycle with ambient temperatures of $23-24^{\circ} \mathrm{C}$. Both HDT and N-HDT rats lost $2-7 \%$ of their body weights during the first 3 days of suspension, but by day 7 they had regained or surpassed their starting weights. Presuspension body weights in HDT $(208 \pm 5.4$ g) and N-HDT rats $(210 \pm 6.6 \mathrm{~g})$ were comparable to 7 -day values $(215 \pm 5.7$ and $211 \pm 7.9$ in HDT and $\mathrm{N}$ HDT rats, respectively). Pentobarbital sodium $(6 \mathrm{mg} /$ $100 \mathrm{~g}$ ) was used to anesthetize rats for cannulation $1 \mathrm{wk}$ before suspension. In each animal a polyethylene catheter (PE-10) was surgically placed in the right carotid 
I 
artery, with the opening at the level of the aortic arch (confirmed at autopsy). All the polyethylene catheters were sterilized using ethylene oxide gas (Anprolene-73) for $12 \mathrm{~h}$. Surgical instruments were maintained in a hospital instrument germicide (Amerse, Vestal Laboratories, St. Louis, MO) before use. Each catheter was exteriorized by passage under the skin to a dorsal position between the scapulae. The aluminum back brace of our WBS apparatus (7) provides a rigid structure for stabilizing and protecting the catheter. Each catheter was flushed with a sterile solution of lithium heparin in physiological saline $(1: 1,000)$ before recording. In the first series of experiments, blood pressures and HRs were determined on days 1, 3, and 7 of HDT and N-HDT suspension and after several hours of recovery ( $\geq 2 \mathrm{~h}, \leq 7 \mathrm{~h}$ ) on day 8. This period was selected for recovery measurements to avoid a potentially rapid redistribution of body fluids after removal from suspension and to allow the rats to resume normal behavioral patterns and posture after reambulation. A saline-filled catheter was connected to a Statham-Gould pressure transducer (model P23), with the diaphragm positioned at heart level in all animals. All recordings were made between 1200 and 1600 (except for the recovery postsuspension rats) in unanesthetized and undisturbed rats in secluded animal quarters. To minimize the potential circadian effects, measurements were made at almost the same time each day. Each rat served as its own control, and presuspension recordings within its vivarium cage were made for each animal. During the recovery experiments, each animal was removed from the suspension apparatus and permitted to move about freely in its own cage; after 2-7 $\mathrm{h}$, recordings were taken. Because the values of individual animals overlapped and did not differ markedly over the 5-h recovery period monitored, the results were combined.

In a second series of experiments, rapid head-up tilt was imposed in each rat after 1 and 3 days of HDT or $\mathrm{N}$-HDT suspension. Three 5-min periods were used: pretilt, tilt, and posttilt measurements were made in the same animal for $5 \mathrm{~min}$ (pretilt) and continued during a vertical $\left(70-90^{\circ}\right)$ head-up tilt. After 5 min, animals were quickly returned to their original HDT or N-HDT positions, and recordings were again made for $5 \mathrm{~min}$ (posttilt). In all animals and at all times, the transducer was positioned at heart level. The aluminum back brace permits a rapid manual rotation, and the rat can be mechanically fixed in the vertical, horizontal, or tilted position. Recordings were made minute by minute for each animal for a 5-min period in each position. Individual as well as averaged values are presented for each period. Recordings were made between 1000 and $1700 \mathrm{~h}$. Data from each group of experiments were tested by analysis of variance for multigroup comparisons. When a statistical difference was noted, treatment significance was determined using a post hoc Student $t$ test, and differences were considered significant at $P<0.05$.

\section{RESULTS}

Seven days of suspension and recovery. HDT resulted in significant elevations of MAP on days 1,3 , and 7 com-
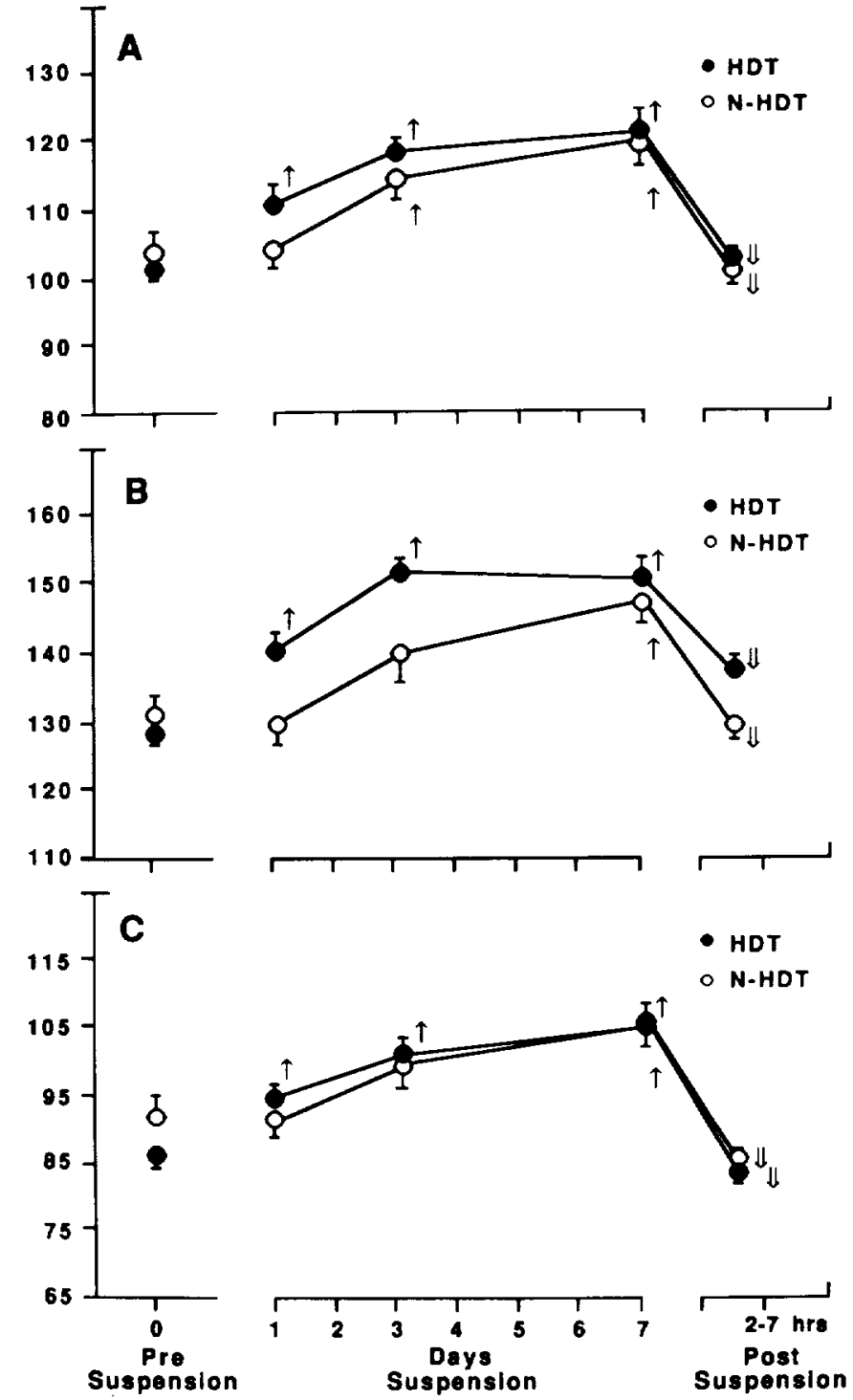

FIG. 1. Blood pressure recordings from 9-13 head-down-tilted (HDT) and 4-10 non-head-down-tilted (N-HDT) rats. Values are means \pm SE for mean arterial pressure $(A)$, systolic pressure $(B)$, and diastolic pressure $(C)$. Significant $(P<0.05)$ elevation from presuspension $(\uparrow)$ or decrease from 7 -day suspension $(\Downarrow)$.

pared with presuspension values (Fig. $1 A$ ). When HDT rats were returned to normal ambulatory activity, the MAP fell to presuspension levels within several hours (Fig. 1A). The N-HDT rats showed some slight, but statistically insignificant, rise in MAP on day 3 and a significant rise on day 7 . Within hours after removal from suspension, the MAP of N-HDT rats also returned to control levels. Within each group there were no significant differences between days 3 and 7 , and there appears to be a plateauing of the MAP response (Fig. $1 A$ ).

The SP (Fig. $1 B$ ) showed a pattern similar to the MAP (Fig. $1 A$ ), with elevations in HDT rats on days 1 and 3 and a sustained elevation on day 7 . Recovery to presuspension levels was also evident after removal from the suspension apparatus, with SP not significantly different from control. In the N-HDT rats, the SP was significantly elevated only on day 7 , with a marked fall to presuspension levels during the period of reambulation. 
TABLE 1. Pulse pressure and heart rate in HDT and N-HDT rats: presuspension (control), suspension, and postsuspension (recovery)

\begin{tabular}{lcc}
\hline \hline & $\begin{array}{c}\text { Pulse Pressure, } \\
\text { mmHg }\end{array}$ & $\begin{array}{c}\text { Heart Rate, } \\
\text { beats } / \text { min }\end{array}$ \\
\hline Presuspension & & \\
HDT (13) & $41.8 \pm 1.8$ & $417 \pm 11.2$ \\
N-HDT (6) & $40.2 \pm 1.6$ & $426 \pm 18.0$ \\
Suspension & & \\
Day I & & \\
HDT (13) & $44.9 \pm 1.6$ & $447 \pm 12.9^{*}$ \\
N-HDT (4) & $37.0 \pm 3.0 \dagger$ & $474 \pm 29.4^{*}$ \\
Day 3 & & \\
HDT (11) & $50.0 \pm 3.6^{*}$ & $458 \pm 11.6^{*}$ \\
N-HDT (4) & $39.3 \pm 4.4$ & $464 \pm 9.5^{*}$ \\
Day 7 & & \\
HDT (10) & $44.2 \pm 2.2$ & $440 \pm 8.2^{*}$ \\
N-HDT (7) & $41.0 \pm 2.1$ & $465 \pm 14.9^{*}$ \\
Postsuspension, 2-7 h & & \\
HDT (9) & $48.8 \pm 3.5^{*}$ & $484 \pm 8.4^{*}$ \\
N-HDT (9) & $39.3 \pm 1.4 \dagger$ & $469 \pm 8.0^{*}$ \\
\hline
\end{tabular}

Values are means $\pm \mathrm{SE}$, with no, of rats in parentheses. HDT, headdown-tilted rats; N-HDT, non-head-down-tilted rats. Significant difference $(P<0.05)$ vs. presuspension control rats $\left({ }^{*}\right)$ or vs. HDT rats $(\dagger)$.

There were significant differences between the two suspension groups on days 1 and 3 (Fig. $1 B$ ).

The DP in HDT rats was also significantly elevated from presuspension levels on days 1, 3, and 7 of suspension (Fig. $1 C$ ). In the N-HDT rats the only significant increase in DP occurred after 7 days. There was a return of DP to presuspension levels after removal from WBS in both HDT and N-HDT rats (Fig. 1C).

Pulse pressures (PP) showed a significant elevation from presuspension levels on day 3 of HDT. Compared with the 7-day rats, there was no significant difference (Table 1). However, the postsuspension value of $48.8 \pm$ $3.5 \mathrm{mmHg}$ was significantly higher than the presuspension value, $41.8 \pm 1.8 \mathrm{mmHg}$. In the $\mathrm{N}$-HDT rats there were no significant changes of PP during any of the periods of measurement. There were significant differences in PP between the two suspension groups on day 1 and during recovery (Table 1 ). HRs were significantly increased throughout the measurement periods in both groups of suspended rats, and this response persisted after removal from suspension (Table 1).

Rapid head-up tilt. Comparisons of the acute responses of HDT and N-HDT rats to rapid head-up tilting are presented in Figs. 2-6. After suspension for 1 day but before tilting, both HDT and N-HDT rats showed significantly elevated $(P<0.05) \mathrm{MAP}$ and SP but not DP compared with presuspension control animals (Figs. 2-4). With a rapid head-up tilt the MAP, SP, and DP showed immediate elevations in both HDT and N-HDT in 1day-suspended rats. In each case, during the $5 \mathrm{~min}$ of head-up tilt after the initial elevation, there was a tendency for the blood pressures to return toward pretilt levels. Returning the animal to its original HDT or NHDT position resulted in significant $(P<0.05)$ falls in MAP, SP, and DP. This was evident in both the minuteby-minute change and the averaged values (Figs. 2-4).

After suspension for 3 days, the pretilt HDT rats responded with highly significant $(P<0.05)$ rises in MAP,

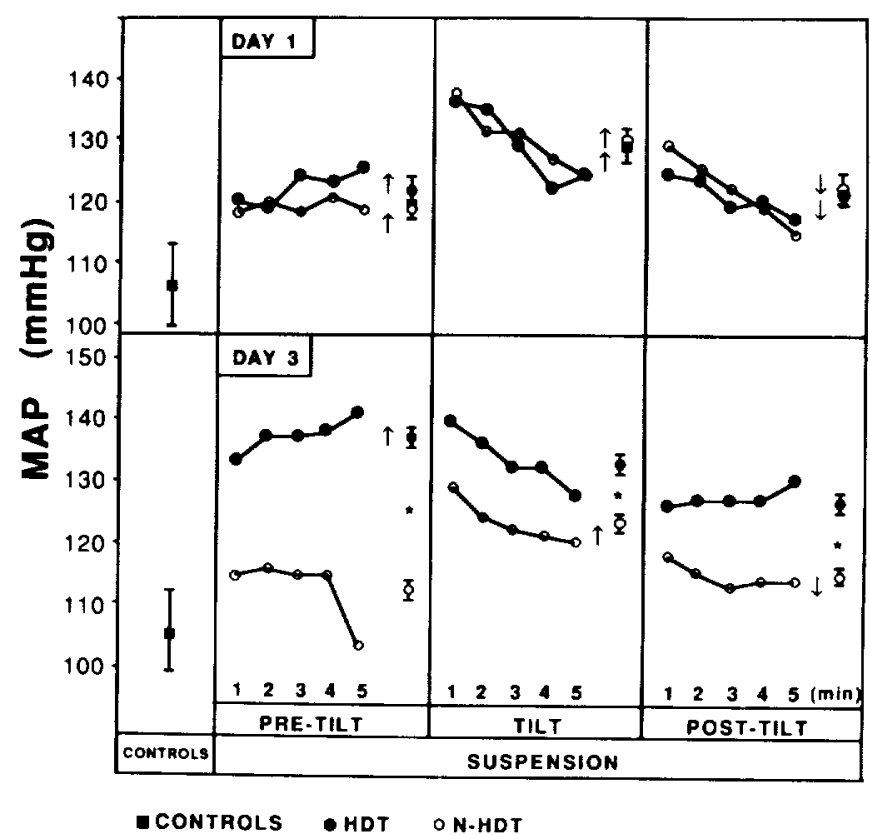

FIG. 2. Mean arterial pressure (MAP) response during rapid headup tilting in rats after 1 and 3 days of suspension in either HDT or $\mathrm{N}$-HDT position. Values are means for each minute and averaged values (means $\pm \mathrm{SE}$ ) for total of $5 \mathrm{~min}$ in each of 3 periods. C. Presuspension (controls), $n=13$ rats; $\bullet$ HDT, $n=5-10$ rats; O, N-HDT, $n=5-6$ rats. Significant difference $(P<0.05)$ from each preceding group ( 1 or $\downarrow)$ and between HDT and N-HDT (*).

SP, and DP (Figs. 2, 3, and 4, respectively) compared with presuspension values. In response to rapid head-up tilt in HDT rats, there was a significant $(P<0.05)$ fall from the pretilt in the averaged MAP values (Fig. 2); the fall in averaged SP was not significant, and the DP showed no change (Fig. 4). In the pretilted N-HDT rats the MAP, SP, and DP values were significantly $(P<$ 0.05 ) lower than those in HDT rats (Figs. 2-4), and only

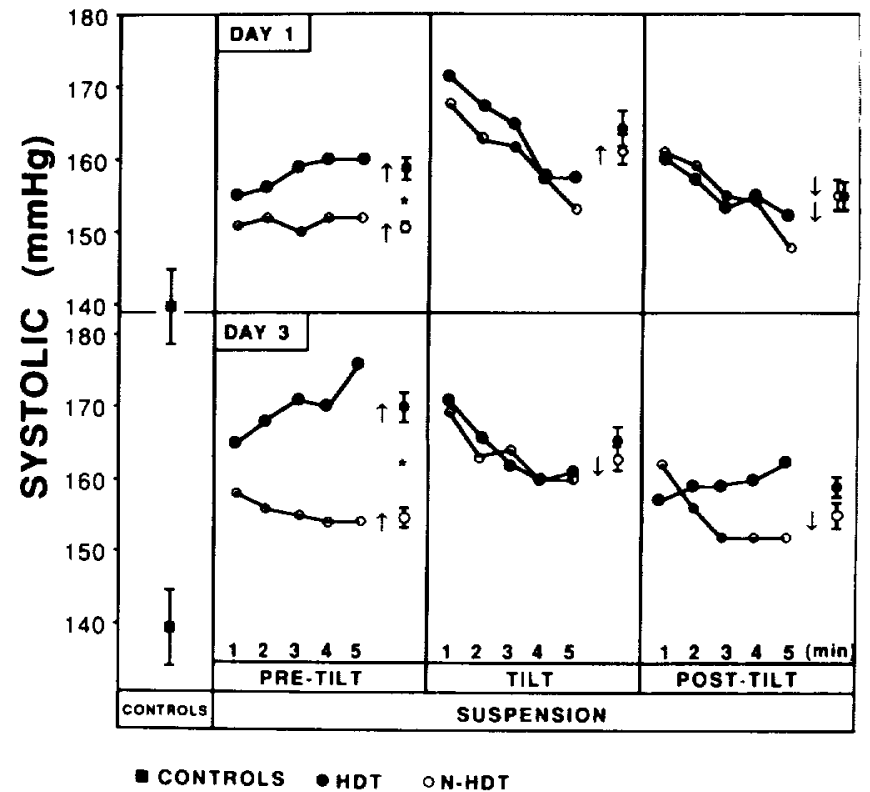

FIG. 3. Systolic pressure response during rapid head-up tilting in rats after 1 and 3 days of suspension in either HDT or N-HDT position. Values are means for each minute and averaged values (means \pm SE) for total of $5 \mathrm{~min}$ in each of 3 periods. Symbols as in Fig. 2 . 


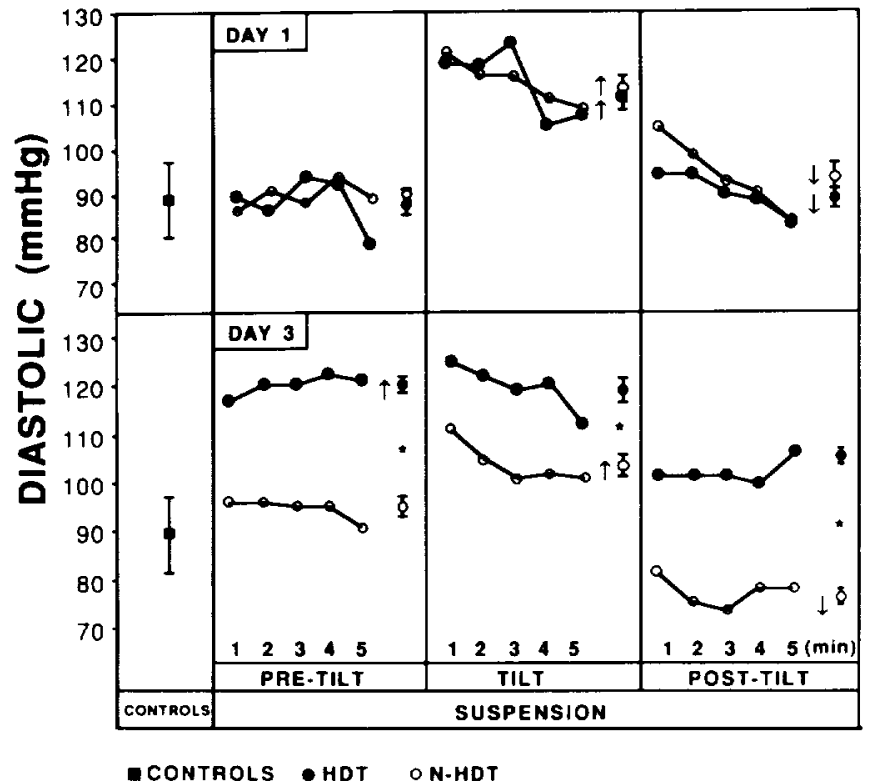

FIG. 4. Diastolic pressure response during rapid head-up tilting in rats after 1 and 3 days of suspension in either HDT or N-HDT position. Values are means for each minute and averaged values (means $\pm \mathrm{SE}$ ) for total of $5 \mathrm{~min}$ in each of 3 periods. Symbols as in Fig. 2.

the SP values were significantly $(P<0.05)$ higher than the presuspension control values (Fig. 3). In the HDT and N-HDT rats, the averaged SP values were comparable during the rapid head-up tilting (Fig. 3). Posttilt return of rats to HDT and N-HDT positions showed that averaged MAP, SP, and DP remained significantly $(P<$ $0.05)$ elevated in the HDT rats compared with presuspension values (Figs. 2-4). However, in the N-HDT rats there was a marked fall in DP in posttilted rats below presuspension levels (Fig. 4).

With 1-day suspensions, PP in HDT and N-HDT rats were not significantly altered from presuspension levels in the pretilt, tilt, and posttilt positions (Fig. 5). How-

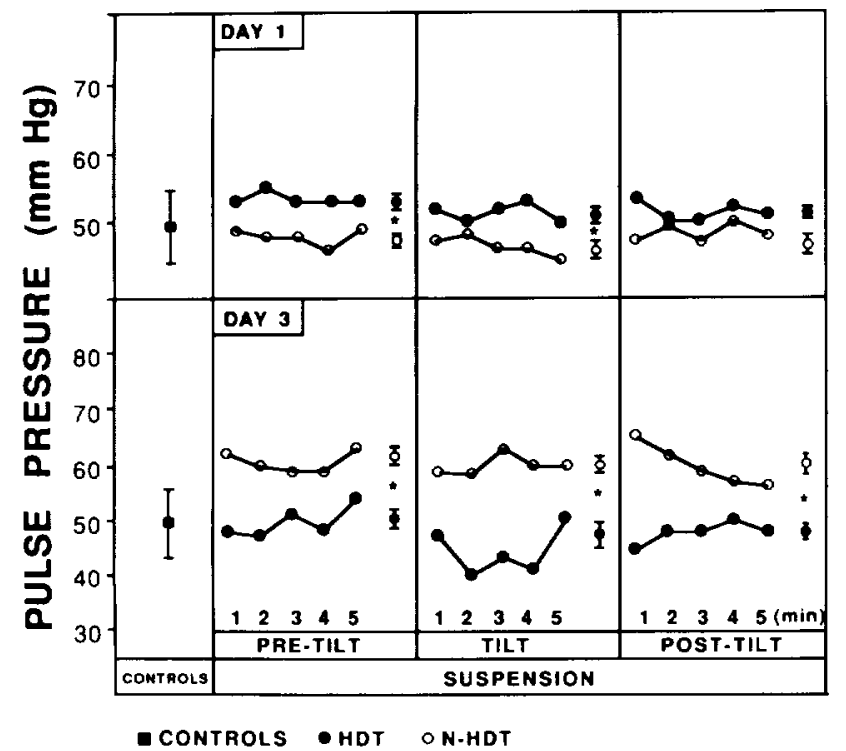

FIG. 5. Pulse pressure response during rapid head-up tilting in rats after 1 and 3 days of suspension in either HDT or N-HDT position. Values are means for each minute and averaged values (means $\pm \mathrm{SE}$ ) for total of $5 \mathrm{~min}$ in each of 3 periods. Symbol as in Fig. 2 .

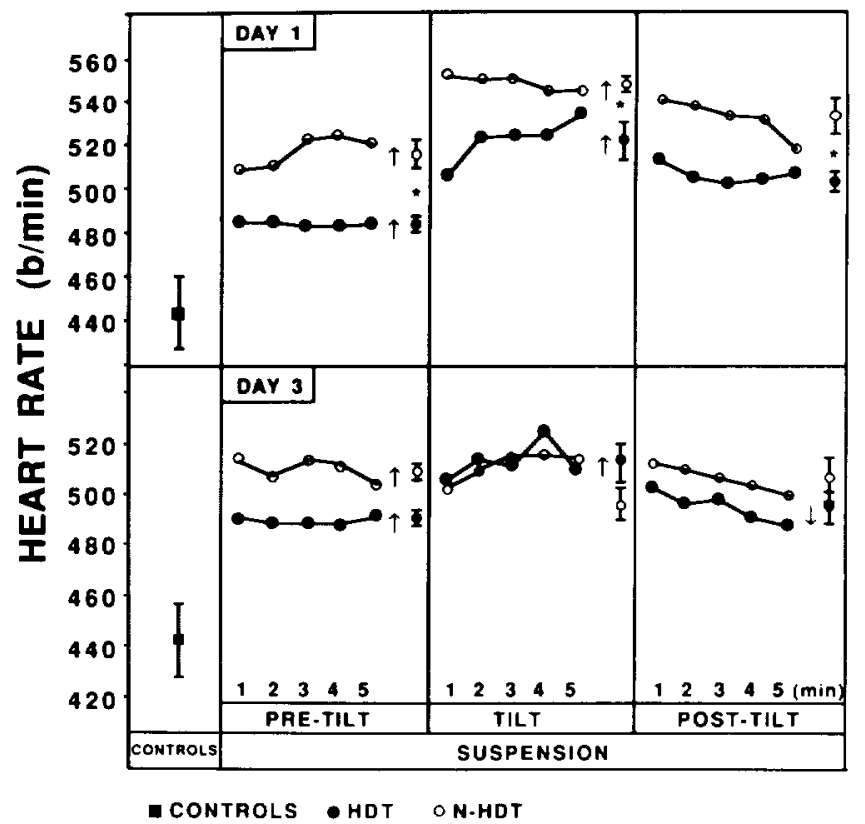

FIG. 6. Heart rate response during rapid head-up tilting in rats after 1 and 3 days of suspension in either HDT or N-HDT positions. Values are means for each minute and averaged values (means $\pm \mathrm{SE}$ ) for total of $5 \mathrm{~min}$ in each of 3 periods. Symbols as in Fig. 2.

ever, the PP were consistently lower in N-HDT than in HDT rats (Fig. 5). After 3 days of suspension, the PP showed wide differences (Fig. 5). The N-HDT animals appeared to be more responsive, with elevations of $\mathrm{PP}$ in all three periods, whereas the HDT animals had lower PP but they were not significantly different from presuspension values (Fig. 5). The HRs in both HDT and N-HDT rats, compared with presuspension rats, were significantly $(P<0.05)$ elevated after 1 day in all stages of positioning (Fig. 6). Although both groups showed significant elevations in $\mathrm{HR}$ after tilting, the N-HDT rats showed a greater tachycardia (Fig. 6). After 3 days of suspension, although HR values of HDT and N-HDT rats were comparably elevated during pretilt, only the HDT showed an increased HR with tilting (Fig. 6). Both the HDT and N-HDT rats showed reversals toward presuspension values during posttilting.

\section{DISCUSSION}

Rats suspended for 7 days in the HDT position responded with elevations in blood pressures. Compared with N-HDT rats these changes are more rapid, starting on day 1 of suspension. When HDT and N-HDT rats were further challenged by rapid head-up tilting on day 1 of suspension, they responded comparably with anticipated increases in blood pressures. However, by day 3 of suspension the two groups responded to rapid head-up tilting in a markedly different manner, with the HDT rats not exhibiting the increased blood pressure noted in $\mathrm{N}$-HDT rats. It should be noted that neither cardiac output nor blood volume was determined in the present study, parameters that could influence the pressure responses reported in the present work. However, Popovic (13) previously reported cardiac output alterations of $\leq 10 \%$ during 1 wk of suspension in unanesthetized rats. 
In addition, Dunn et al. (4) noted no significant alterations in total blood volume, red cell mass, or plasma volume when these were expressed relative to body weight in rats suspended for 7 days. Therefore the role of changes in cardiac output and vascular volume in the blood pressure responses reported for suspended rats would appear to be limited.

Long-term exposure of humans to microgravity (27-84 days during Skylab missions) was reported to increase resting HR and SP and decrease MAP and DP (6). Shorter duration human exposures (2-8 days aboard the Space Shuttle), more comparable to the time frame of the present experiments, elicited increases in both HR and MAP (2). Results of the first series of experiments in the present study are consistent with these latter observations, suggesting that the WBS model can simulate the cardiovascular alterations induced by microgravity exposure. Bed rest (both horizontal and HDT) in humans has also been utilized as a ground-based simulation of the effects of microgravity. Short-term (24-h) HDT bed rest is not associated with alterations in resting supine HR, SP, or DP (11). Longer duration (3-wk) bed rest was associated with a relative tachycardia but no change in MAP (1).

Popovic (13) used a suspension system with chronic cannulations of the aorta in 7-day HDT and recovery. He reported that the MAP was decreased $(\sim 20 \mathrm{mmHg})$ during the first 2 days of suspension, followed by an elevation to normal values $(115-120 \mathrm{mmHg}$ ), which remained unchanged during recovery. Popovic (13) also reported a slight rise in HR during the first 2 days of HDT, a return to control levels during the remaining period of suspension, and a significant elevation during the initial 3 days of recovery. His suspension HR data are not in agreement with the current results in either of the present experiments. Although the HR in our rats started at a slightly higher level, $\sim 415-425$ beats $/ \mathrm{min}$, there was a significant increase on day 1 and a continued elevation through day 7 (Table 1). In the HDT rats, there was a clear and significant rise during the early hours of recovery, a response comparable to that of Popovic (13).

One assumption has been that in HDT rats there is a cephalad fluid shift (5). Shellock et al. (15), Stump et al. (17), and Tucker et al. (18) demonstrated that functional changes, whether cardiovascular or renal, are supportive of this reasoning. However, it remains unclear that there is a cause-and-effect relationship between changes in blood pressure and the fluid shift in the rat WBS model. Although there are some similarities between primates and rodents in response to HDT, such as diuresis, natriuresis, and tachycardia, questions remain concerning conclusions that may be drawn from such comparisons.

Passive head-up tilting of humans to $70^{\circ}$ from the supine position and lower body negative pressure (LBNP) of $-50 \mathrm{mmHg}$ produce elevations of $\mathrm{HR}$, an immediate reduction of SP, but DP changes in the opposite direction in the two models (19). Reflex increases in peripheral arterial resistance produce an increase in MAP when head-up tilt is maintained (1). Cardiovascular deconditioning (orthostatic intolerance) in humans is evidenced after microgravity exposure by an exaggerated HR increase in response to passive head-up tilting (2) or LBNP
(6). Whereas in-flight LBNP resulted in increased DP and MAP and lower SP and PP (6), postflight passive tilting (2) resulted in a reduction of MAP. Our experiments with rapid head-up-tilted rats were initiated with the hypothesis that, if the HDT rat is a suitable model for deconditioning, then reversal of the cephalad fluid shift by tilting should produce characteristics of orthostatic intolerance, including an exaggerated HR increase, decreased MAP, and perhaps syncope. The observation that rapid head-up tilting during day 1 of HDT suspension induced a marked elevation in blood pressures was inconsistent with a rapid deconditioning hypothesis. To some extent both HDT and N-HDT rats showed comparable tendencies in response to rapid tilting on day 1 of suspension. The absence of an elevated MAP in the 3day-suspended HDT rats, in contrast to the significant increase in N-HDT animals, suggests that characteristics of deconditioning may be developing. This is consistent with a significantly lower PP during tilting on day 3 of suspension in HDT compared with N-HDT rats. However, the HR response to tilting in HDT rats is not exaggerated compared with the N-HDT group. Therefore, tilting experiments in the HDT rat provide inconsistent evidence for deconditioning responses. Utilizing LBNP and administration of sympathomimetic drugs, Overton and Tipton (12) previously reported little evidence for deconditioning responses in suspended rats.

The stability of PP and lack of sensitivity to rapid head-up tilt during day 1 of HDT and N-HDT show a relative level of adaptation. On day 3 , although there is a significant reversal in PP between the HDT and N-HDT rats, the rapid head-up tilt maneuver did not elicit any significant response in HDT rats. This lack of response to rapid head-up tilting suggests that these animals may have adjusted to the transient hypertension due to HDT. However, the fact that HR was elevated in both suspension postures suggests that confinement or restricted movements may be more responsible for the tachycardia.

Popovic et al. (14) relate that their animals "struggle" to escape from the harness and that this may contribute to a slightly altered cardiac output. It must be emphasized that animals used in this study did not struggle, and those few that showed any initial reluctance to adapt to the suspension system were eliminated during the first hours of day 1. Extreme care was taken to record in undisturbed quiet animals to obtain data that more accurately reflect their position. Although we attempted to reduce stress to minimal levels, there is some consensus that stress-related features are evident in suspension models $(10,14)$.

Several conclusions may be drawn from these two series of experiments. The WBS model does demonstrate several cardiovascular alterations consistent with those observed after microgravity exposure. MAP and HR increases in HDT rats in the first series of experiments are consistent with flight results. These responses were also evident in N-HDT aniamls but were slower to develop, suggesting that perhaps stress in addition to positioning contributes to the cardiovascular alterations. Passive head-up tilting studies of the WBS rat suggest the potential for the development of cardiovascular deconditioning, but not all responses are consistent with this conclu- 
sion. The rat model may be an adequate surrogate for humans in many areas of space research, including muscle atrophy, bone resorption, and fluid and electrolyte excretion; however, additional more extensive and mechanistic studies of cardiac responses are required to definitely characterize the suspension model as an adequate ground-based simulation for cardiovascular adaptation to microgravity. Studies extending over longer durations of WBS, coupled with determinations of fluid shifts and measurements of plasma renin activity during passive tilting, would be of value in further characterizing the validity of the WBS model as a simulation of microgravity effects on the cardiovascular system.

This work was supported by National Aeronautics and Space Administration Grant NAG 2-386, a grant from the Southern Regional Education Board, and the University of Louisville President's Incentive Fund.

Animal experimentation was conducted in accordance with the document "Guiding Principles in the Care and Use of Animals," approved by the Council of the American Physiological Society, and the protocol was approved by the Institutional Animal Use and Care Committee.

Address for reprint requests: J. M. Steffen, Dept. of Biology, University of Louisville, Louisville, KY 40292.

Received 11 March 1991; accepted in final form 26 April 1992.

\section{REFERENCES}

1. Blomqvist, C. G., AND H. L. Stone. Cardiovascular adjustments to gravitational stress. In: Handbook of Physiology. The Cardiovascular System. Peripheral Circulation and Organ Blood Flow. Bethesda, MD: Am. Physiol. Soc., 1983, sect. 2, vol. III, pt. 2, chapt. 28 , p. 1025-1063.

2. Bungo, M. W., J. B. Charles, ANd P. C. Johnson. Cardiovascular deconditioning during space flight and the use of saline as a counter-measure to orthostatic intolerance. Aviat. Space Environ. Med. 56: 985-990, 1985.

3. Deavers, D. R., X. J. Musacchia, And J. A. Meininger. A model for antiorthostatic hypokinesia: head-down tilt effects on water and salt excretion. J. Appl. Physiol. 49: 576-582, 1980.

4. Dunn, C. D. R., P. C. Johnson, R. D. Lange, L. Perez, and R. NESSEL. Regulation of hematopoiesis in rats exposed to antiorth. ostatic, hypo-kinetic/hypodynamia. I. Model description. Aviat. Space Environ. Med. 56: 419-426, 1985.

5. Hargens, A. R., J. Steskal, C. Johansson, and C. M. Tipton.
Tissue fluid shift, forelimb loading, and tail tension in tail suspended rats. Physiologist 27, Suppl.: S37-S38, 1984.

6. Johnson, R. L., G. W. Hoffler, A. E. Nicogossian, S. A. BergMAN, AND M. M. JACKSON. Lower body negative pressure: third manned Skylab mission. In: Biomedical Results from Skylab, edited by R. S. Johnston and L. F. Dietlein. Washington, DC: NASA, 1977 , p. $284-312$.

7. MUSACCHIA, X. J., AND D. R. DEAVERS. A new rat model for studies of hypokinesia and antiorthostasis. In: Aduances in Physiological Sciences. Gravitational Physiology, edited by J. Hideg and O. Gazenko. New York: Pergamon, 1981, vol. 19, p. 149-152.

8. Musacchia, X. J., D. R. Deavers, J. A. Meininger, and T. P. Davis. A model for hypokinesia: effects on muscle atrophy in the rat. J. Appl. Physiol. 48: 479-486, 1980.

9. Musacchia, X. J., AND J. M. StefFen. Short term (1 and 3 day) cardiovascular adjustments to suspension antiorthostasis in rats. Physiologist 25, Suppl.: S163-S164, 1982.

10. Musacchia, X. J., AND J. M. Steffen. Cardiovascular and hormonal (aldosterone) responses in a rat model which mimics responses to weightlessness. Physiologist 27, Suppl.: S41-S42, 1984.

11. Nixon, J. V., R. G. Murray, C. Bryant, R. L. Johnson, JR., J. H. Mitchell, O. B. Holland, C. Gomez-Sanchez, P. VergneMARINI, AND C. G. BLOMQVIST. Early cardiovascular adaptation to simulated zero gravity. J. Appl. Physiol. 46: 541-548, 1979.

12. OverTon, J. M., AND C. M. TIPTON. Effect of hindlimb suspension and cardiovascular responses to sympathomimetic and lower body negative pressure. J. Appl. Physiol. 68: 355-362, 1990.

13. Popovic, V. Antiorthostatic hypokinesia and circulation in the rat. Physiologist 24, Suppl:: S15-S16, 1981.

14. Popovic, V., P. PoPovic, AND C. HoneycutT. Hormonal changes in antiorthostatic rats. Physiologist 25, Suppl:: S77-S78, 1982.

15. Shellock, F. G., H. J. C. Swan, and S. A. Rubin. Early central venous pressure changes in the rat during two different levels of head-down suspension. Aviat. Space Environ. Med. 56: 791-795, 1985.

16. Stump, C. S., J. M. Overton, AND C. M. Tipton. Influence of single hindlimb support during simulated weightlessness in the rat. J. Appl. Physiol. 68: 627-634, 1990.

17. Tipton, C. M., J. M. Overton, M. J. Joyner, and A. R. HarGENS. Local fluid shifts in humans and rats: comparison of simulation models with actual weightlessness. Physiologist 30, Suppl: S117-S120, 1987.

18. TuCker, B. J., C. A. Mundy, M. G. Ziegler, C. BAylis, AND R. C. Blantz. Head-down tilt and restraint on renal function and glomerular dynamies in the rat. J. Appl. Physiol. 63: 505-513, 1987.

19. Wolthuis, R. A., S. A. Bergman, AND A. E. Nicogossian. Physiological effects of locally applied reduced pressure in man. Physiol. Rev. 54: 566-595, 1974. 
